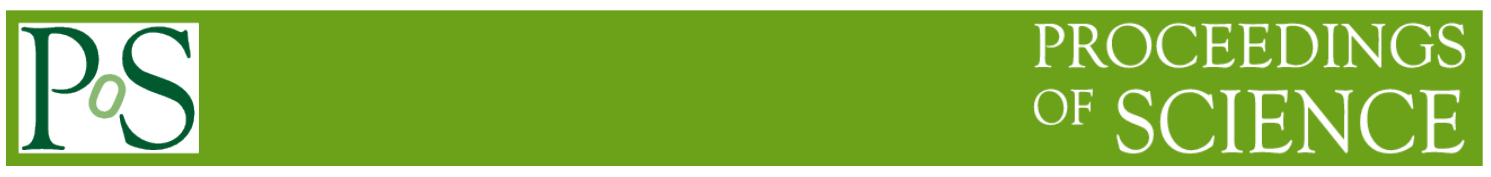

\title{
Cosmology with the WSRT
}

\section{Hans Rudolf de Ruiter}

INAF, Istituto di Radioastronomia, Via Gobetti 101, 40129 Bologna, Italy

E-mail: deruiter@ira.inaf.it

\section{George Miley}

Leiden Obs., Fac. Science, University Leiden, Postbus 9500, 2300 RA, Leiden, The Netherlands E-mail:miley@strw.leidenuniv.nl

\section{Michael Garrett}

School of Phys. \& ASTRON, Alan Turing Bldg., University of Manchester, Oxford Road, Manchester M13 9PL, UK

E-mail: michael.garrett@manchester.ac.uk

50 Years Westerbork Radio Observatory, A Continuing Journey to Discoveries and Innovations Richard Strom, Arnold van Ardenne, Steve Torchinsky (eds) 


\title{
Cosmology with the WSRT
}

\section{Chapter 11.1 Radio Continuum Surveys and Cosmology}

Hans Rudolf de Ruiter*

\begin{abstract}
A the time the Westerbork Synthesis Radio Telescope started observing, 4 in June 1970, the memory of a vivacious and sometimes bitter dispute 1 Lamong astronomers was still fresh. This debate had to do with one of the most profound philosophical questions: what is the origin and the structure of the Universe? An important group of astronomers, among whom the famous Fred Hoyle, Hermann Bondi and Geoffrey Burbidge, had developed the "Steady State" cosmology, as an alternative for the "Big Bang" (a term coined by Hoyle, intended to be disparaging). In the "Steady State" view the Universe had always existed and would exist, unchanging, forever. Radio astronomy turned out to play a fundamental role in the eventual confirmation of the big-bang cosmology as the most favoured theory of the origin of the Universe. In the late fifties and early sixties astronomers started to map the radio sky. English has a very handy word for this kind of activity: survey. It is amusing to think that we would have used the German word "Durchmusterung" if radio astronomy had been developed a hundred years earlier, when stellar catalogues were constructed like the Bonner Durchmusterung. Somehow the $3 \mathrm{C}$ Durchmusterung sounds a bit odd...
\end{abstract}

Radio source counts in the first radio surveys (e.g. the $3 \mathrm{C}$ ) caused a great deal of excitement, because it was immediately clear that the number of fainter sources was more than one would have expected in a static universe. Many of the radio sources were associated with galaxies and with "radio stars". Maarten Schmidt established in 1963 that the latter objects had high redshifts and were thus presumably located at large distances. Certainly, they were not normal stars in our galaxy. Maarten Schmidt coined the term quasars (from quasi-stellar objects) for them. They were quickly interpreted as the active nuclei of galaxies, in which highly energetic processes produce enormous quantities of energy, expelled in the form of matter and electromagnetic radiation. Gigantic black holes in galactic nuclei are the only feasible way of causing such energetic events.

* IRA-INAF Bologna, Italy

The radio source counts, together with the discovery of the cosmic microwave background, were so compelling that the Big Bang model was now generally accepted. 
Radio astronomy had contributed to a breakthrough in cosmology, making it possible to study the evolution of known and perhaps unknown classes of radio source populations (galaxies and quasar and objects still to be discovered).

Such was the state of affairs when the WSRT entered the stage. A serious problem had always been the rather limited accuracy of radio source positions. Redshifts can almost exclusively be measured in the optical range of the electromagnetic spectrum thanks to the presence of well-known spectral lines characteristic of elements like hydrogen, oxygen, and so on. Since a radio source by itself does not give information on the distance, precise positions are necessary to know what, or rather where, exactly you are looking at. The new generation of interferometric radio telescope arrays, in particular the WSRT, could deliver a positional accuracy that was sufficient for this purpose: the WSRT was a unique and revolutionary instrument in the decade between 1970 and 1980 . Even after the advent of the VLA, around 1980, the cosmological studies based on the WSRT remained important. The distribution of tasks between the two Dutch universities involved in WSRT observations, Groningen and Leiden, turned out to be very successful and fertile. Groningen specialized in neutral hydrogen observations (the famous $21 \mathrm{~cm}$ spectral line of hydrogen) and would produce ground-breaking results.

Leiden concentrated on continuum studies (both surveys and individual galaxies). The dynamic Prof. Harry van der Laan supervised a number of PhD students-including myself-doing this kind of cosmological study, during the 1970 and 80 s. A selection of some of the theses is given at the end.

A harbinger was Jet Katgert-Merkelijn, who got her PhD degree in 1970 (see Thesis [a]). She determined the radio luminosity function of radio galaxies, using the Australian Parkes telescope. Her work was the beginning a series of similar studies at Leiden Observatory.

Among the first persons carrying out continuum radio surveys, right after the inauguration of the WSRT, were Rudolf Le Poole and Peter Katgert (Thesis [b]). They spent much time to get to know the WSRT properties, the calibration and the software, which helped successive observers in understanding in detail how to treat the WSRT data.

In 1973, Claas Oosterbaan, Tony Willis and I started to work with data obtained with the WSRT. Our aim was to construct a catalogue of faint radio sources and identify them optically with galaxies and quasars. We therefore tried to use optical plates that could detect the faintest possible objects. By the way, this was long before the now universally present CCD detectors had been invented.

Two years later Tony Willis and I spent a few months in Pasadena, because we could use the beautiful optical material owned by Halton C. Arp, an astronomer who worked at the Mount Wilson and Palomar Observatories. Arp had a vast archive of thousands of optical plates; most of these (Kodak) plates used the then hypermodern IIIa-J emulsion, which was capable of registering very faint objects.

The demise of the steady-state theory was hard to swallow for some. Although the support for it dwindled to a trickle, a number of hard-core adversaries of the Big Bang now started to question the cosmological nature of quasars; in particular, they maintained that the redshifts of quasars were not an indication of their distances, unlike the redshifts of galaxies.

I was only vaguely aware that Arp was perhaps the most vociferous of the "dis sidents", who were against the common interpretation of quasar redshifts. Arp would stick to his convictions right until his death in 2013. Arp had a pleasant and open character, but I quickly found out that he had a quasi-emotional problem with statistics, not so much with the mathematics but with the general acceptance of certain concepts like probability and likelihood. Although I investigated some of his claims in detail later on, I could see no reason at all to abandon the conventional view of the redshift as an indicator of distance.

The optical identification of radio sources, that is, finding optical counterparts at the radio position, was a laborious, time-consuming process, especially com pared to the modern computer-based cross-correlations between radio, optical, X-ray or any other wavelength data sets. Once you have the data sets available, linking these sets is a matter of seconds, even if many thousands of objects are involved. Tony Willis, Claas Oosterbaan and myself-see Thesis [c]-carried out a survey of radio sources, found as background sources in observations that were done for specific sources, mostly nearby radio galaxies. This is, by the way, the reason why there was so much optical material collected by Arp: he was interested in bright and possibly disturbed galaxies many of whom had been observed with the WSRT as well.

Jet Katgert-Merkeliin ${ }^{1}$ gave, on behalf of the people who had done surveys with the WSRT in the early days, a summary of the work done so far, and presented this at the IAU Symposium 74 (1977). I reproduce her table in Figure 1.

Of course, there is not enough space here to discuss the many, many studies based on all the WSRT surveys done in the past years. The analysis of the cosmological evolution of power and size of radio sources is beyond the scope of this short article.

The positional accuracy of the WSRT turned out to be very well matched with the optical material like the III a-J plates. As shown in Figure 1 about one third to one quarter of the radio sources had an optical counterpart, often an elliptical galaxy, or a candidate quasar. The true nature of the latter had to be confirmed later by spectroscopical means. The deep plates were far superior to the Paloma Sky Survey, but did not cover the whole (northern) sky. Tony Willis ${ }^{2}$ (always a the same IAU Symposium 74) summarized the WSRT radio continuum surveys. 


\begin{tabular}{|c|c|c|c|c|c|c|}
\hline Authors & $\begin{array}{l}\text { Freq. } \\
\text { (MHz) }\end{array}$ & $\mathrm{N}$ & $\mathrm{S}_{(\mathrm{imJ})}$ & Plates & $\mathrm{m}_{1 \mathrm{im}}$ & $\begin{array}{l}\text { ID } \\
\%\end{array}$ \\
\hline De Ruiter et & 1415 & 462 & $3-9$ & III a-J & 22.5 & 27 \\
\hline a1., 1976 & & 39 & $6-9$ & III a-J & 24.0 & 49 \\
\hline Katgert \& Spin- & 1415 & 53 & 7 & PSS & 20.5 & 26 \\
\hline $\mathrm{rad}, 1974$ & & 32 & 7 & $098-02$ & 22.0 & 43 \\
\hline Jaffe \& Perola, & 1415 & & $4-5$ & III $a-j$ & 22.5 & \\
\hline $1975 \delta>20$ & & 40 & & & & 28 \\
\hline$\delta<20$ & & 34 & & & & 29 \\
\hline $\begin{array}{l}\text { Katgert et a1., } \\
1973\end{array}$ & 1415 & 166 & $6-10$ & PSS & 20.5 & 18 \\
\hline Valentijn et & 610 & $\begin{array}{l}88 \\
38\end{array}$ & $\begin{array}{r}6 \\
16\end{array}$ & III a-J & 22.5 & 35 \\
\hline $\begin{array}{l}\text { al., } 1977 \\
\text { Katgert, } 1977\end{array}$ & 610 & $\begin{array}{r}38 \\
226\end{array}$ & $16^{16}-29$ & PSS & 20.5 & $\begin{array}{l}26 \\
17\end{array}$ \\
\hline
\end{tabular}

Figure 1 WSRT continuum surveys until 1977. Although many surveys used an observing frequency of $1415 \mathrm{MHz}$, some were done at $610 \mathrm{MHz}$. Limiting flux densities were typically in the range 1-10 mJy (with modern telescopes and detectors we can easily go down to a thousand times fainter fluxes). The optical data normally used came from the Palomar Sky Survey but the much more sensitive III a-1 had become widely available (see columns 5 and 6 ). Also given is the percentage of radio sources for which an optical counterport had been found. Roughly two thirds of the sources remained unidentifed, presumably because the optical objects was sa faint as to be below the pate linit (see the identifcation percentage in column 7 ). Note that the strength or brightess is expressed in $m / y$ (millijansky), which is equal to.

Since all surveys covered only small parts of the northern sky, very few of the strongest sources (which are quite rare), were present, and one finds sources only below $1 \mathrm{Jy}$, which happens to be at the maximum of the normalized source counts (see Figure 2). For historical reasons the source counts are "normalized"

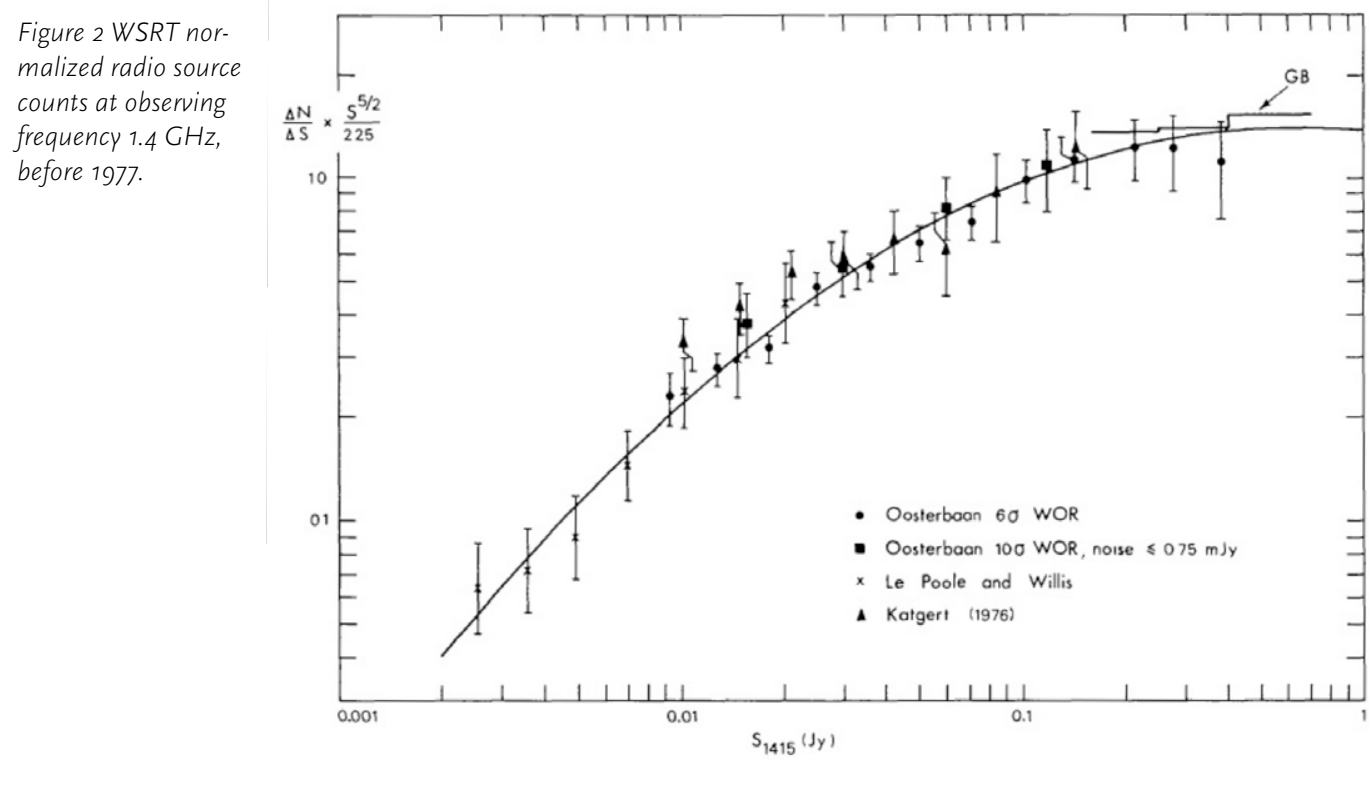

with what is called the Euclidean count. This is actually much simpler to understand than it might appear. Counting the sources found and dividing them in intervals of strength (flux density) the resulting distribution is divided by the one expect if the sources were uniformly distributed. As seen in Figure 2 the WSRT could cover the range between $1 \mathrm{mJy}$ and 1 Jy. Not seen (as there are no strong sources above $1 \mathrm{Jy}$ ) is the part of the source count that was the death blow to the steady state theory. The shape of the counts for fainter sources is exactly what you would expect: it not a horizontal line due to the expansion of the universe and other well-known cosmological effects.

In a way, our 1970-1980 Universe was reassuringly simple. "Dark matter" and "dark energy" were unknown, even though there were some early indications of dark matter, as Fritz Zwicky had pointed out already in the 1930s. We had stars and stellar systems (which were called collectively galaxies), and presumed that quasars were nothing but the highly active nuclei of galaxies. In such a nucleus we imagine that a gigantic black hole is lurking, spitting out energy in various forms (like for example radio waves). An active galactic nucleus often enters the literature under its acronym AGN and this could comprehend both galaxies and quasars. Much theoretical work followed and a qualitative model consisting of a central black hole emitting double-sided radio jets, which might travel to enormous distances from the nucleus (up to many millions of light years), was accepted by most astronomers. In addition, the mechanism of the radio radiation (synchrotron emission) was known in detail since the 1950s.

The first studies about samples of radio sources dealt with their composition: how many galaxies were present? How many were quasars? Did the ratio change going to fainter sources? It became clear that bright (optically as well as in the radio) quasars must have evolved strongly: in early stages of the universe, there were far more of such bright quasars than at the present epoch. This was about the extent of our knowledge in the early 1970s. The PhD theses of Jet Katgert-Merkelijn, Peter Katgert, and myself (see Figure 3), tried to give an inventory of the then known populations of radio sources, their luminosity

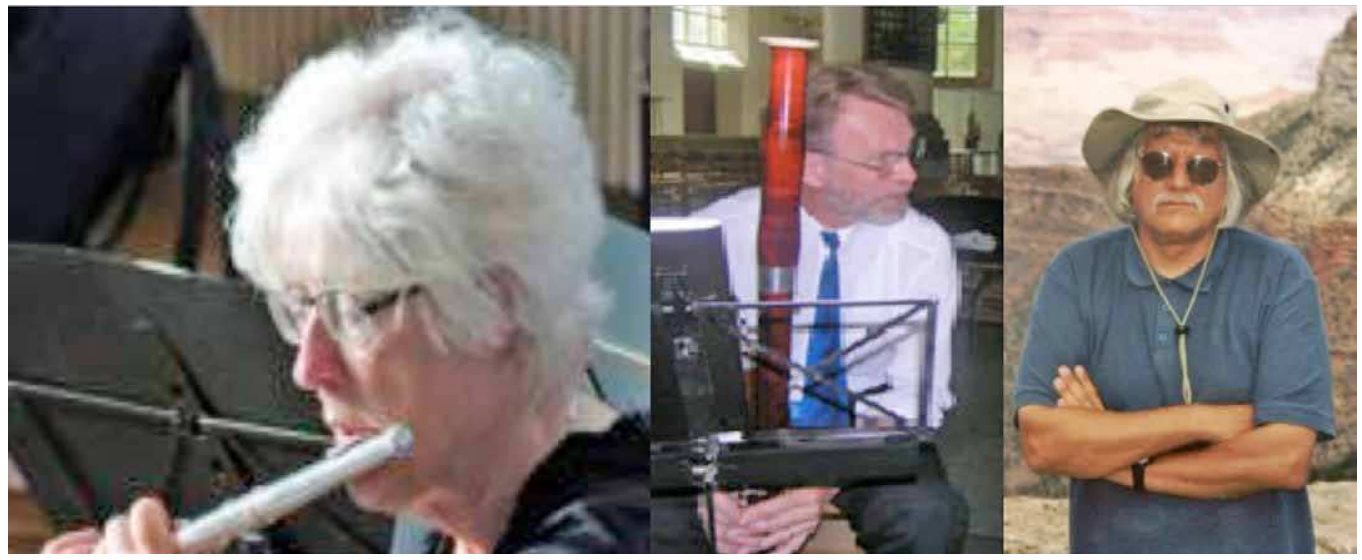


functions (that is the distribution of their radio powers) and the cosmological evolution of the various constituents like elliptical radio galaxies and quasars).

Perhaps the most serious limitation of these early studies was the lack of spectroscopic data. Since the redshift of spectral lines is the only way to determine the distance, only some of the most nearby and brightest galaxies and quasars had spectral data available. Fortunately, radio galaxies were often found in the brightest and most massive elliptical galaxies, these had always very similar optical luminosities. For that reason, one could get a rough estimate of their distance notwithstanding the lack of spectral data.

Strong radio sources associated with such elliptical galaxies and with quasars in general were found to evolve very strongly in the sense that they were much more common in the early epochs of the Universe. These studies based on indirect methods were very uncertain and only in recent years there is significant progress in the collection of spectroscopic data, thanks to important projects like the SDSS (Sloan Digital Sky Survey).

It would take another 5-10 years, before a new batch of $\mathrm{PhD}$ students (Windhorst, Thesis [d], and Marc Oort, Thesis[e]), would explore the sub-mJy level (o.1-1 mJy). A simple model of a hypothetical population of objects could easily reproduce the shape of the radio source counts, as had been demonstrated by Longair already in the 1960 s, if one includes cosmological evolution of the population. At lower flux levels, also non-evolving or only mildly evolving population should be present, like for example spiral galaxies, but these would enter our sample only below $1 \mathrm{mJy}$ and perhaps even much lower.

Figure 4 Rogier Windhorst
and Marc Oort.
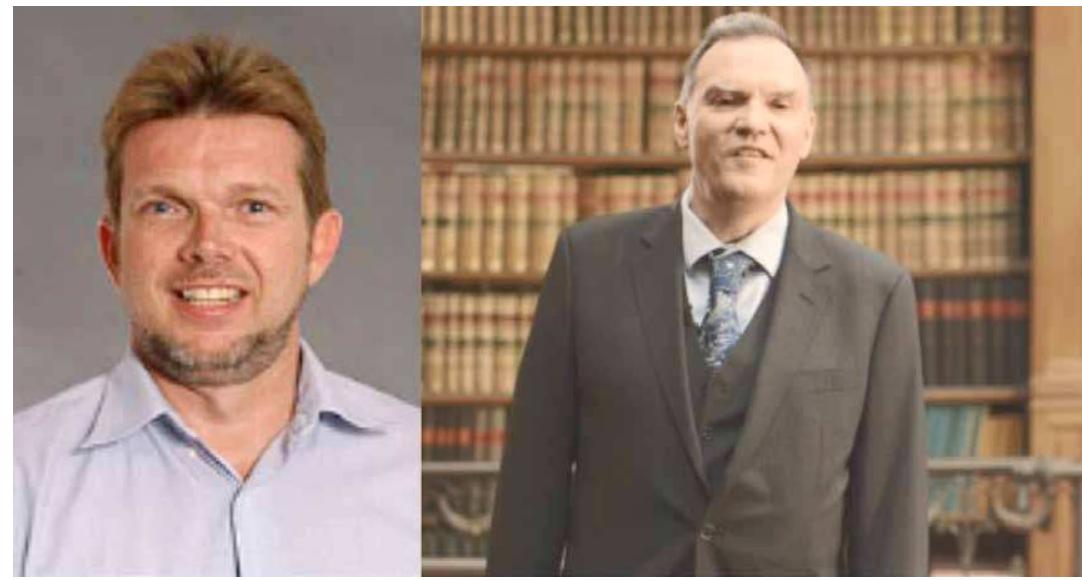

From 1980 onward, the WSRT carried out some deep surveys, and these were studied by Rogier Windhorst and Marc Oort (see Figure 4). Their work would lead to "definitive" source counts at $1.4 \mathrm{GHz}$, down to $0.1 \mathrm{mJy}$, in large part based on WSRT observations.
The pictures (Figures 3 and 4) of the five Leiden PhD students are more recent than their PhD theses..

The most important and beautiful figure is, no, not Figure 3 or 4 , but Figure 5 taken from an article by Rogier Windhorst ${ }^{3}$ and his collaborators. There you can see in every detail all the properties of the radio source counts starting at about $0.1 \mathrm{mJy}$ (100 times fainter than in the studies of the 1970s!) going upwards to the strongest sources at $10^{4} \mathrm{mJy}$ or more. VLA and Cambridge surveys confirmed the results of Windhorst and Oort.

Note that the counts at the high end (at about 1000 mJy, corresponding to 3 in the logarithm) go down again towards the brightest flux densities. In Figure 2 strong sources were absent due to their paucity.

Perhaps equally interesting is the flattening of the source counts that starts just above a mJy and becomes more pronounced towards the faint end at 0.1 $\mathrm{mJy}$. Windhorst and later Oort found that a new (in the sense of a hitherto unknown) population of blue radio galaxies was responsible for the flattening of the counts, and not the usual red elliptical galaxies, which abound at the higher flux densities together with the quasars.

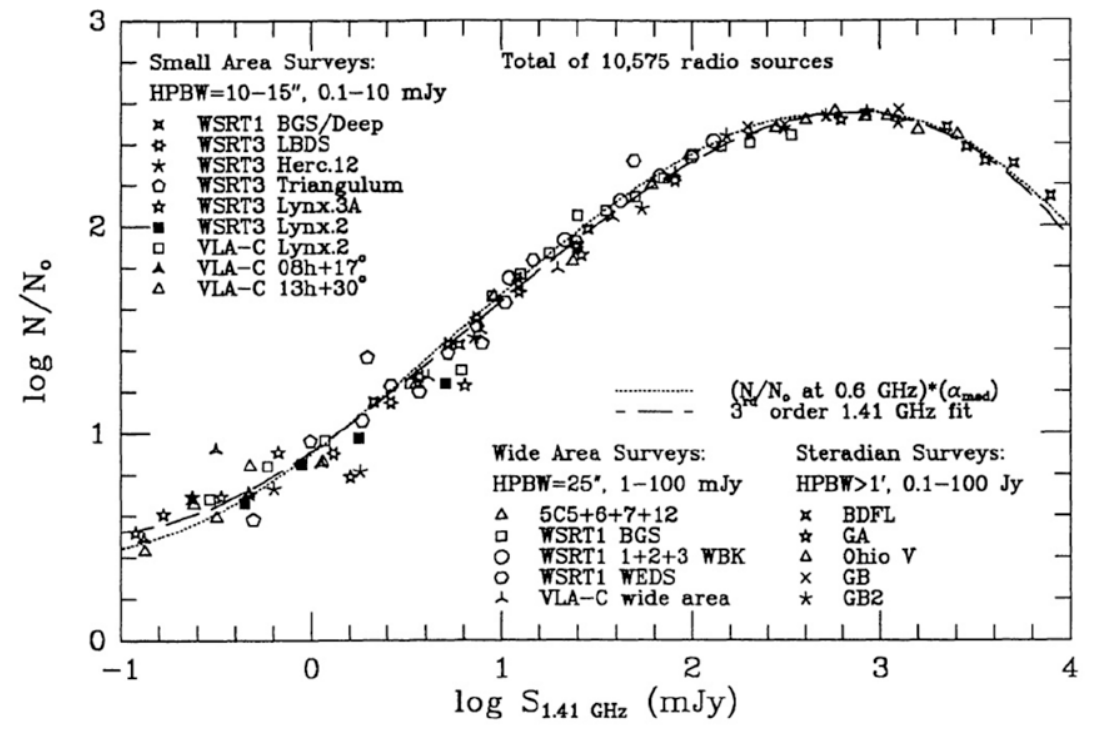

It remains unclear, even until the present day, what these galaxies really are, but most astronomers consider them to be "starburst", or star forming, galaxies. The radio emission is then not, at least not predominantly, due to an active nucleus, but to an anomalously strong burst of star formation. Of course, a phase of strong activity in the galaxy nucleus may indirectly have caused strong star formation, a possibility that shows how simple and naïve our ideas about source populations were: reality may be much more complex.
Figure 5 The normalized definitive radio counts at $1.4 \mathrm{GHz}$. Note the flattening below $1 \mathrm{~m} / \mathrm{y}(\log \mathrm{S}=$ $0)$, which is due to a new population of 
There may be sub-populations of radio galaxies, which sometimes partly overlap. Classifying them all may be a hard task. We find a bewildering zoo of different classes of objects in modern literature. For example, Padovani ${ }^{4}$ gives the following list: FSQ (flat spectrum quasars), SSQ (steep spectrum quasars). FRI (Fanaroff_Riley type I radio sources), FRII (type II radio sources), BL Lacs (a kind of quasar-like objects without clear spectral lines), radio-quiet AGNs, and elliptical galaxies with active star formation. It is by no means clear how and to what extent all these different but partly overlapping classes of objects contribute to the source counts. In any case we know that the presence of an Active Galactic Nucleus may or may not be accompanied by strong star formation (and vice-versa)

Below 1 mJy star forming galaxies start to appear, but the classical AGNs we see among the strongest radio sources still make up about $50 \%$ of the radio sources. This may even be so at $\mu \mathrm{Jy}$ levels, i.e. at levels a thousand times fainter than the mJy sources. De Zotti ${ }^{5}$ and collaborators discuss this in detail. Considering the confusion that still reigns it is wise to keep the models as simple as possible, for the moment at least.

Very deep surveys (going down to a few $\mu \mathrm{Jy}$ ), were carried out mainly with the VLA, but even the WSRT (with its modern instrumentation) can reach these levels. The improvement in sensitivity by a factor of about 1000 in about 30 years is impressive indeed. A number of studies were done with the VLA, in the region of the Hubble Deep Field (HDF). Worth mentioning are the studies by Richards et al. ${ }^{6}$ at $8.5 \mathrm{GHz}$ down to a limit of $9 \mu \mathrm{Jy}$, Fomalont et al. ${ }^{7}$ at 8.5 $\mathrm{GHz}$ down to $7.5 \mu \mathrm{Jy}$ and Richards ${ }^{8}$ at $1.4 \mathrm{GHz}$ with a limit of $40 \mu \mathrm{Jy}$. The WSRT cannot stand up to such a firepower, one might say, but this is only partly true.

In the same period, a group of astronomers led by former ASTRON director Mike Garret ${ }^{9}$ made very deep observations with the WSRT of the HDF region, which reached the unprecedented level of $40 \mu \mathrm{Jy}$, without too much effort. The observing time (six 12-hour periods) was very similar to the times used for the extensive surveys in the early 1970s. In Figure 6, we show the field observed by the WSRT in the form of a contour plot.

Since the planning of the biggest radio telescope ever, the Square Kilometer Array (SKA) has started, modelling radio source counts down to very faint levels (nano Jansky-a thousandth of a $\mu \mathrm{J} y$ ) has become a popular pastime. Many articles have discussed the possible populations that might be present and at what level they would enter the counts. All astronomers appear to agree about one thing: if you arrive at the level of 1 nanoJy, you have seen virtually everything there is to see. In fact, Condon et al. ${ }^{10}$ assert that at that level about 95 $\%$ of all the radiation, originating in known types of radio sources, as briefly sketched above, is detected. The remainder should come from the billions of stars, planets (whether populated by aliens or not) and perhaps some extremely weak populations of sources we have no inkling about (yet). Indeed, some in-

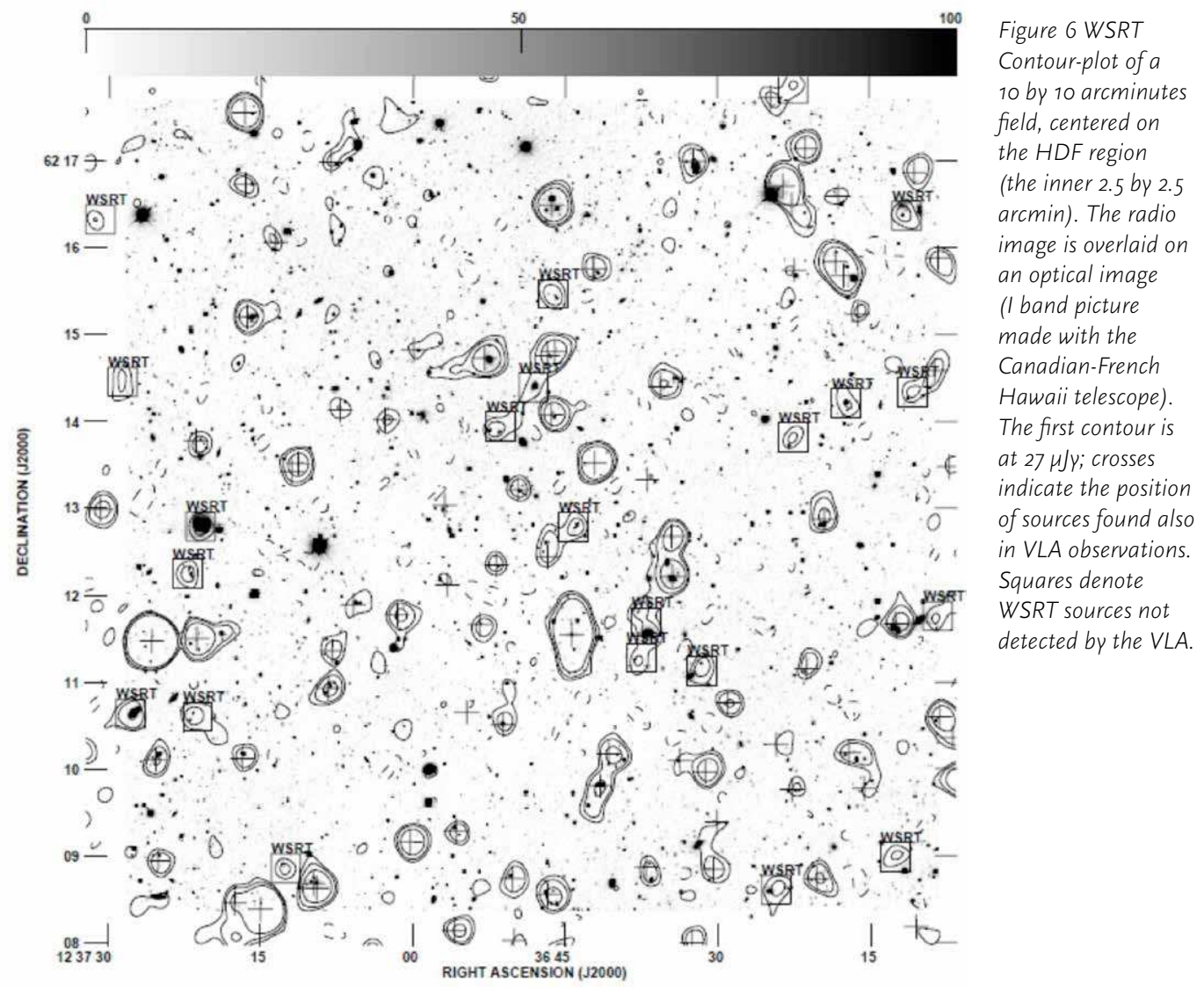

direct evidence points to the latter possibility. It turns out that the temperature of the extragalactic background radiation in the range $3-90 \mathrm{GHz}$ is higher than one would expect based on the known populations of radio sources (ARCADE 2 measurements, see Fixsen et al." ${ }^{11}$. Condon ${ }^{10}$ concludes from this that there is room for some new, still unknown populations. Since real data at the nanoJy level are lacking, his conclusion is obviously pure speculation, but no doubt, there is lots of interesting work to be done with SKA.

Now that we have entered the era of "overwhelmingly large" telescopes, fast computers and terabytes of memory even in small PCs, astronomy has started to produce ever-bigger data sets of objects at all imaginable wavelengths, and surveys have taken on a new meaning. Especially the all sky surveys are all the rage, and the possibility to access such the data sets online is changing astronomical research dramatically. Already more than 20 years ago, a WSRT all sky survey was performed; well, not really all sky, but that part of the sky visible from the far north of the Netherlands. To be even more precise, the part of the sky above a declination of 30 degrees, for which high quality mapping is possible. While the VLA mapped a large part of the sky at $1.4 \mathrm{GHz}$ (FIRST and 
NVSS, both directly accessible from the NED Website, at ned.ipac.caltech.edu), WENSS surveyed the northern sky at lower frequency, $325 \mathrm{MHz}$. WENSS is described in Rengelink et al. ${ }^{2}$.

Anybody, astronomer or non, can easily access Westerbork data, a genuinely democratic operation: just go to Westerbork on the Web (at wow.astron.nl), read the (very simple) instructions and type in the position or the object name and get your own piece of the radio sky at your home computer. As an example I show the field obtained by requesting the data of $3 \mathrm{C}_{23} 8$; the resulting map is given in Figure 7. The file sent to you is in FITS format (used extensively by the astronomical community). The only thing you need is a viewer for FITS files (for example SAOimage, which can be downloaded freely). If you wish, SAOimage can save the FITS file in more common photographic formats like GIF or JPG.

The importance of the modern radio surveys is enormous, not in the least because similar developments in other wavelength ranges (including truly all sky surveys done with satellites, like GALEX-ultra-violet-and 2MASS-infra-red), have revolutionized studies based on the use of these big data sets. The low observing frequency of the WSRT ( $325 \mathrm{MHz}$ ) opens up the possible to hunt for specific types of radio sources, by combining the WSRT with the higher frequency data of the VLA surveys at $1.4 \mathrm{GHz}$, and studying the spectral properties. Wieringa \& Katgert' ${ }^{13}$ used partial 327 and 608 WSRT data (WENSS had not been completed at that time) to select sources with steep spectra, i.e. sources much stronger at the lower frequency. It was known that many sources with steep radio spectra are at very high redshifts, so that selection based on spectrum is an efficient way for finding the most distant sources. This kind of study has been repeated on a much larger scale, by combining all available all-sky surveys (WSRT, VLA, others) and having the computer select the sources with steep spectra. This can be done in a fully automated way, saving time and effort. Carlos de Breuck ${ }^{14}$ and collaborators have done such a study, with great success.

How successful was the cosmological research done with the WSRT? This kind of question is hard to answer of course, and perhaps is rather meaningless. Nevertheless, in my opinion the strategy to mix deep surveys in specific small parts with less deep surveys over larger parts of the sky worked quite well. In the first years one could not reach the sub-mJy level yet, but on the other hand one could take advantage of the simple fact that all WSRT observations contributed "for free" to a background of radio sources in fields observed for quite different purposes. An example is the Background Survey used in my own thesis (Thesis [c]). Much later Windhorst and Oort could concentrate on small fields and dedicated themselves to extended observations at different radio frequencies, which reached levels close to $0.1 \mathrm{mJy}$. Even without the fast computing facilities of today and without the huge databases at optical, infrared, UV and X frequencies existing now, they were able to carry out extended optical observing campaigns. This work turned out to be very successful.

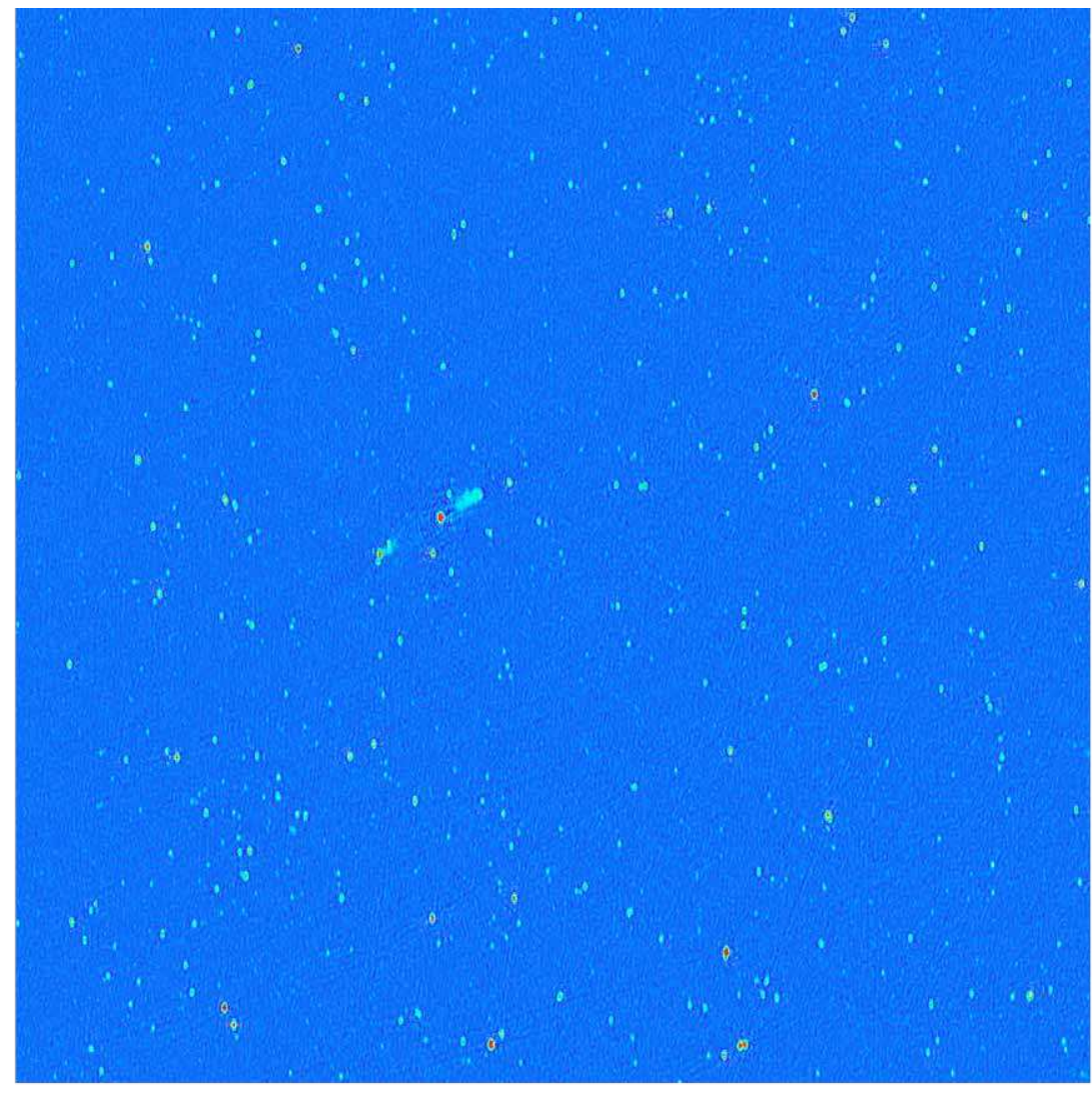

My final opinion is therefore positive. The WSRT has performed a logical sequence of cosmological studies dedicated to the exploration of the various constituents of the early universe and their evolution over time. The possibilities of the WSRT have been exploited as much as was possible in the 1970 s and $1980 \mathrm{os}$ until the role of the WSRT was mostly-but by no means entirely-taken over by the VLA, and Australia Telescope in the Southern Hemisphere. The future cosmological studies with the SKA will be a direct continuation of much of the work for which the WSRT has laid the foundation.

\section{WSRT Surveys: Leiden PhD Theses in the period 1970-1990}

[a] Jet Merkelijn 1970: A determination of the Luminosity Function of radio galaxies at 400 and 2700 $\mathrm{MH}$

b] Peter Katgert 1977: populations of weak radio sources.

Hans de Ruiter 1978: faint extragalactic radio sources and their optical identifications.

Marc Oort 1987: radio galaxies at very low flux levels. 


\title{
Cosmology with the WSRT
}

\section{Chapter 11.2 WSRT observations of ultra- steep spectrum sources}

\author{
Pointing the way to the most massive \\ forming galaxies and clusters and to LOFAR
}

George Miley*

\section{$\mathrm{D}$} istant luminous radio galaxies are unique laboratories for studying the formation of the most massive galaxies and clusters in the early Universe (redshifts $\mathrm{z} \sim 2$ to $\mathrm{z} \sim 5$ ). During its first decade, the WSRT played an important role in laying the groundwork for such studies. This topic was also one of the main scientific applications that motivated the original LOFAR proposal to ASTRON in 1997

Most radio sources detected in low-frequency surveys have "normal" steep radio spectra, with indices $-0.6>\alpha>-0.9$. Only about $10 \%$ of all sources have "ultra-steep" spectral indices with $\alpha<-1.0$. In the early seventies very little was known about these ultra-steep radio (USS) sources. The limited studies that had been carried out with Cambridge telescopes in the sixties indicated that brigh metre-wave USS sources occurred exclusively in nearby rich clusters of galaxies, but the statistics were sparse. In the early seventies Tony Willis and I thought that it would be interesting to use the WSRT to study a significant sample of decametre USS sources from the $4 \mathrm{C}$ Catalogue. The $4 \mathrm{C}$ Catalogue provided a relatively large database for such investigations. In 1973 and 1974 we therefore made several short "cut" observations on $46{ }_{4} \mathrm{C}$ USS sources having USS spectral indices (<1.0 between 178 and $1400 \mathrm{MHz})$. We would compare our results with a similar study that was being carried out on sources with normal radio spectra (Padrielli and Conway et al. 1977, Astron Astrophys. Suppl. 27, 171).

The reduction and analysis was suited for an undergraduate research project and we found a young student who was looking for such a project and was willing to do it. His name was Xander Tielens. Xander later went on to become one of the most important world players in interstellar molecule and come one of the most important world players in interstellar molecule and 


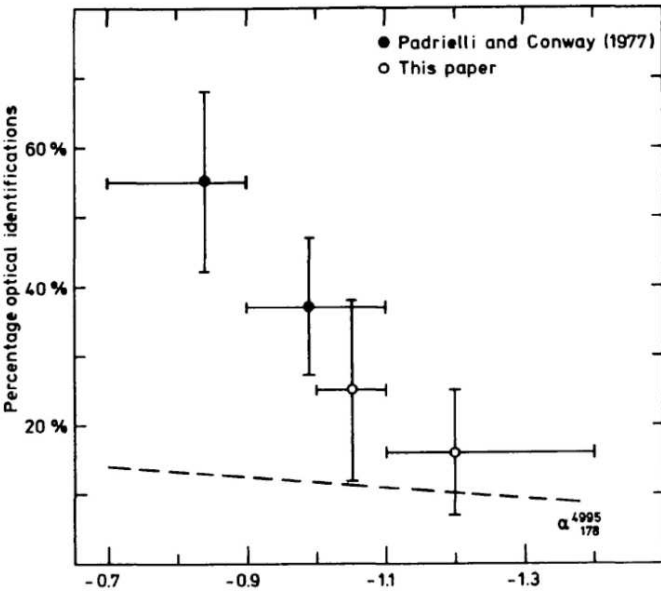

Figure 1. Percentage sources whose WSRT positions resulted in definite optical identifications on the Palomar Sky Survey plotted against spectral index. The plot showed for the first time that radio sources with ultra-steep spectra are located at significantly greater distances than sources with normal spectrum 35. 153-162). When th and Willis, 1978, Astron. Astrophys. Suppl. 35, 153-162). When this paper was published the most distant known radio galaxy was 3 1973. Ap Lett, 216 , 87 - L 8 ). Most of the 4 C USS sources survered by the WVSRT were later found to be associated with massive forming galaxies with redshifts $2<z<5$.

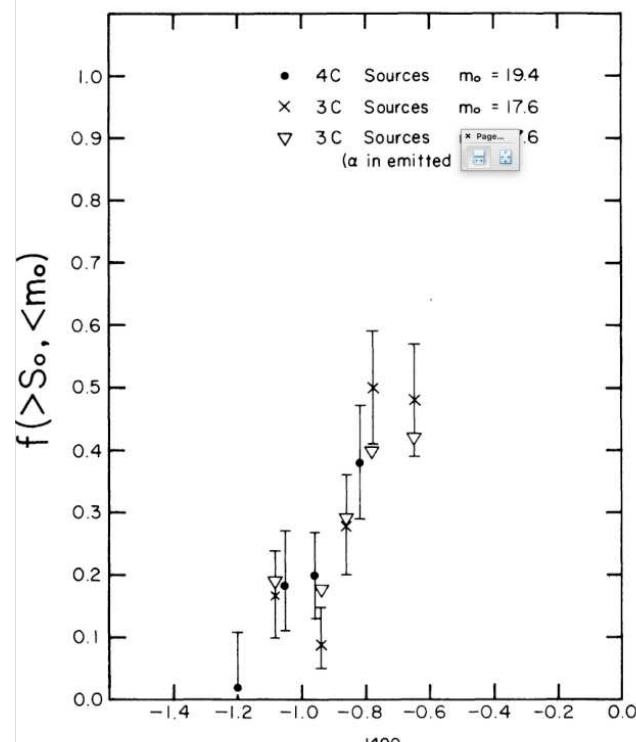

$\alpha_{178}^{1400}$

Figure 2. Fraction of radio sources with counterparts brighter than a given optical magnitude, $m$ plotted against spectral index (Blumenthal and Miley 1979, Astron. Astrophys. 80, 13-21).

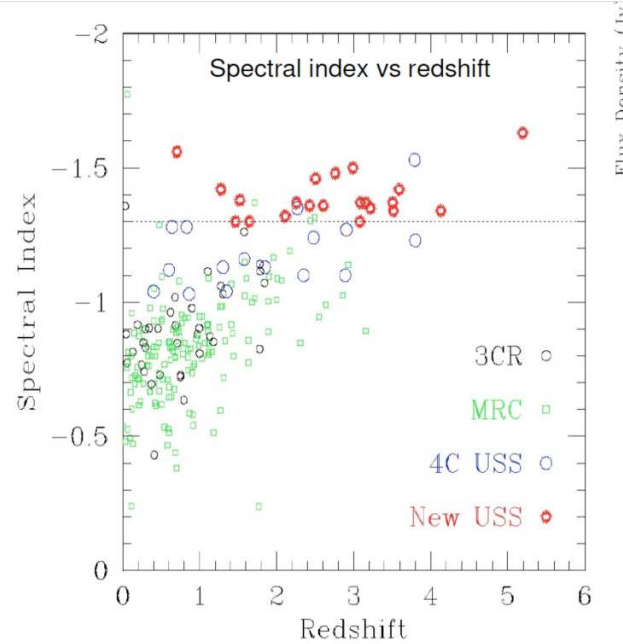

Figure 3. Plot of radio spectral index versus redshift, showing that more distant sources have steeper spectra (De Beuck wan Breugel, Miley Rottgering Stanford and Carilli, 2000, Astron. Astrophys. Supp 143, 303-333)

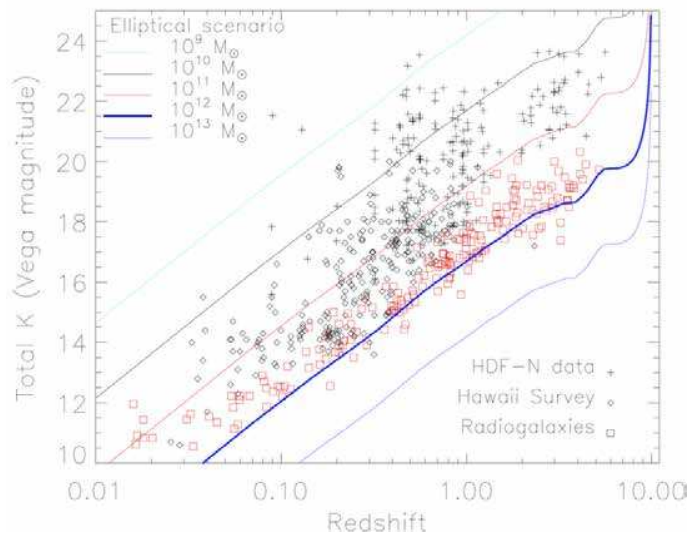

Figure 4. USS radio sources pinpoint the most massive galaxies in the early Universe. Hubble K-z Diagram of radio and optically selected galaxies. Radio galaxies. denoted by the red squares occupy a bright upper enuelope. Prepared by Brigitte Rocca and taken from Miley and De Breuck 2008. Astron. Astrophys. Rev $15,67-144$

sample showed that USS ${ }_{4} \mathrm{C}$ sources differed significantly from normal spectrum 4C sources (Tielens, Miley and Willis, 1978, Astron. Astrophys. Suppl. 35, 153-162). Many more USS sources were unresolved $(<\sim 20$ "), indicating that they were smaller than normal sources. However, the most startling difference was apparent when we tried to identify the USS sources with optical objects on the Palomar Sky Survey. Most of them were blank fields (see Figure 1), indicating that USS sources are located at systematically much greater distances than normal spectrum sources.

While on sabbatical at the U. Cal. Santa Cruz, I looked into the implications of this result with cosmologist, George Blumenthal. Our conclusion (Figure 2) was that USS radio galaxies and quasars followed the same behaviour. From the available data it was difficult to distinguish between a spectral index dependence of radio source luminosity and redshift.

The implications of this result was not followed up until the mid-eighties, when I spent a 4-year leave of absence from Leiden at the Space Telescope Science Institute. The tragic Challenger accident and the postponement of the Hubble

launch created unexpected time for research. I devised a PhD project for Ken Chambers a gifted Johns Hopkins student and contacted Wil van Breugel at the University of California, who had access to the Keck Telescope. We started a programme of optical and infrared imaging and optical spectroscopy of the WSRT USS sample, using any telescope we could get our hands on. The result surpassed our wildest dreams. We broke the distance record for a radio galaxies several times, with objects such as $4 \mathrm{C} 40.36$ at $\mathrm{z}=2.3$ and the monster radio galaxy $4 \mathrm{C} 41.17$ at $\mathrm{z}=3.8$.

The USS detection technique for finding distant radio galaxies developed with the WSRT has since been extremely successful. Most of the several hundred known $\mathrm{z}>2$ luminous radio galaxies were found by targeting small USS radio sources (Figure 3). Massive galaxies and protoclusters detected using the USS technique have been the topic of dedicated ESO Large and Key projects, many observing hours with the Hubble Telecope and intensive study by other large telescopes throughout the electromagnetic spectrum. They have also been food for many Leiden PhD theses, including those of Huub Röttgering, Rob van Ojik, Laura Pentericci, Carlos De Breuck, Roeland Rengelink and Huib Intema. 


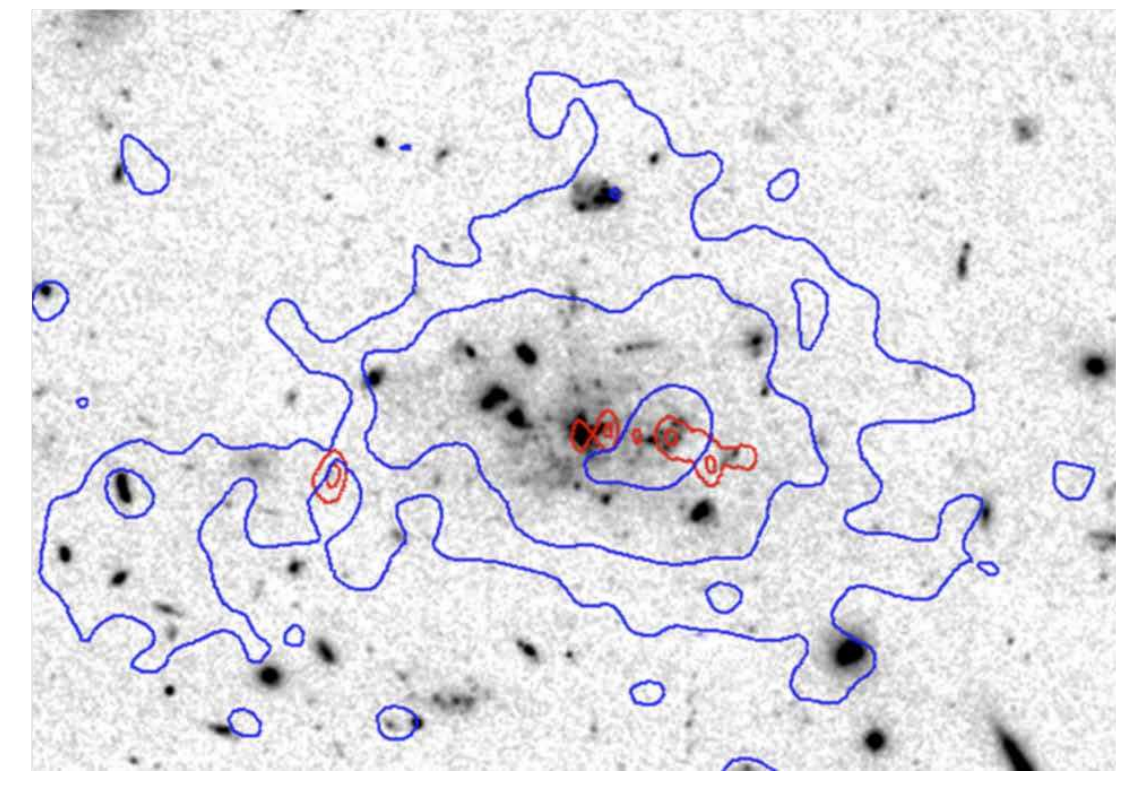

Figure 5. Deep Hubble ACS mage of the Spiderweb Calaxy at $z=2.2$. This is one of the most spectacular distant and massive galaxies found using the WSRT-developed USS technique. The blue contours from the VLT show the giant $150 \mathrm{kpc}$ halo that surrounds it and the red contours from the VLA shows the radio source (Miley et al. 2008, ApJ Letl $\mathrm{C}_{50}, \mathrm{~L}_{2} 9-\mathrm{L}_{32}$ ). The galaxy complex is embedded in a forming protocluster. The Spiderweb and similar objects are excellent laboratories for studying the formation and evolution of the most massive galaxies and clusters and black holes in the early Universe and processes such as galaxy merging, ACN feedback and galaxy downsizing.

A detailed description of the properties of distant luminous radio galaxies and their environment is outside the scope of this contribution (e.g. review by Miley and De Breuck 2008, Astron. Astrophys. Rev. 15, 67 - 144). Their properties indicate that they are the progenitors of brightest cluster galaxies in the local Universe. The various building blocks of high-redshift USS radio galaxies (relativistic plasma, gas in various forms, dust, stars and a powerful AGN) are relatively bright and provide unique diagnostics about the early Universe. The Spiderweb Galaxy (Figure 5) and similar objects are laboratories for studying the formation and evolution of the most massive galaxies and clusters and black holes in the early Universe and processes such as galaxy merging, AGN feedback and galaxy downsizing.

Distant USS radio sources and their "monster" galaxy counterparts also played an important role in motivating the 1997 proposal to ASTRON for a low-frequency array called LOFAR (Figure 6). In the late nineties, the WSRT was almost 30 years old and ASTRON were devoting all their future efforts into developing a do-all mega-facility the Square Kilometre Array Interferometer (now SKA). It was generally expected that SKAI would be finished in $10-15$ years, so the suggestion of building a less ambitious facility to fill the gap was under-

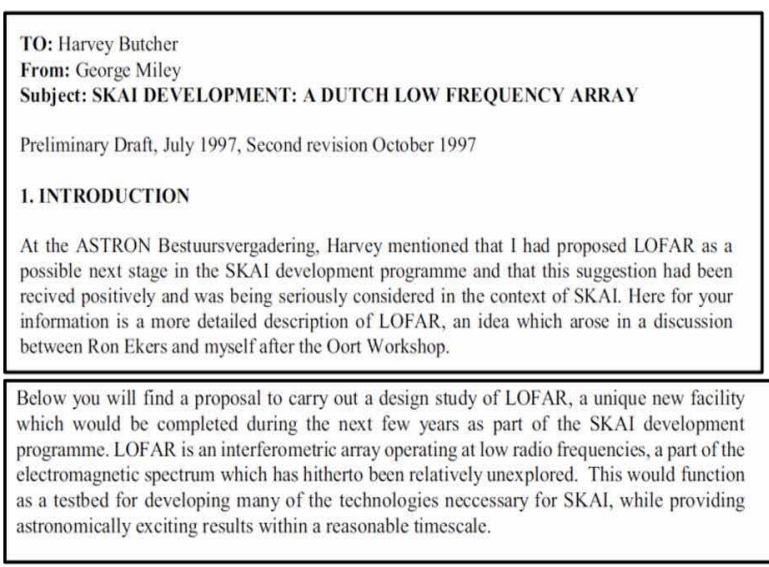
for a low frequency for a low-frequency array called LOFAR. The detection of USS sources at low frequencies and the importance of this for studying forming massive galaxies and clusters in the early Universe was an important motivation for the LOFAR science case.

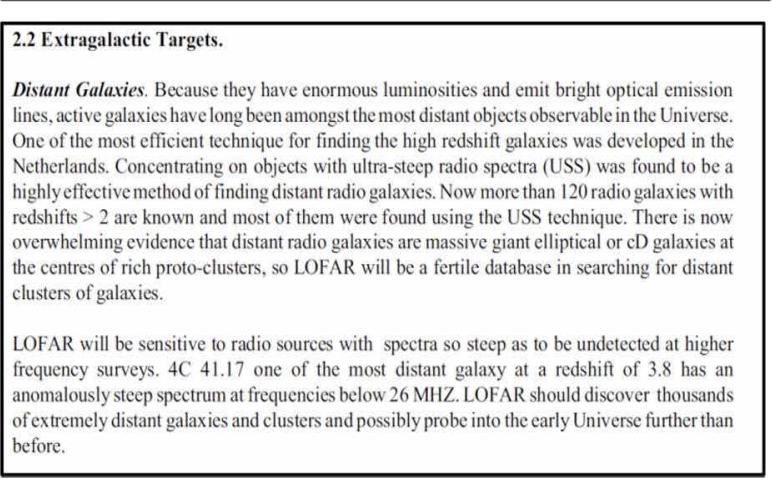

standably not received "with open arms" by everybody concerned, particularly a low-frequency facility in the RFI environment of the Netherlands. However there were other arguments that emerged to justify building a low-frequency facility. First, LOFAR would provide an opportunity to develop several of the techniques that would be needed for SKA and trying them out in a hostile RFI environment. Brilliant ASTRON engineers, such as Jaap Bregman using revolutionary technology, developed LOFAR into a sophisticated software telescope and technology platform. Secondly, Ger De Bruyn became passionately involved in searching for HI emission from the Epoch of Reionisation, that became another prime motivation for LOFAR. Thirdly, Harvey Butcher ensured that LOFAR was more than just a radio telescope. It became a "multidisciplinary sensor array" with geophysical and precision agriculture applications, eligible for ICT funding. Finally, Harvey and Eugene de Geus and the Dutch province of Drenthe conducted a masterly campaign to persuade policy makers that building LOFAR would benefit the Netherlands. The rest is history! 


\section{Cosmology with the WSRT}

\section{Chapter 17.3 WSRT and VLBI observations of the Hubble Deep Field (HDF-N)}

Michael Garrett**

Introduction

In early 1999, the WSRT upgrade was transitioning towards completion. Although the broadband IVC-DZB system was fully available yet, a new suite of very sensitive Multi-frequency Front End (MFFE) receivers were already achieving $\sim 30 \mathrm{~K}$ noise levels on the telescope. Dr. Willem Baan, Radio Observatory director, was looking around for projects that would demonstrate the new capabilities of the WSRT to the international community. Around the same time, I had become very interested in the Hubble Deep Field North (HDF-N) - a small patch of sky only a few arcminutes across, that had been observed by the Hubble Space Telescope (HST) for a then unprecedented integration time of 10 full days! I was not the only astronomer to be very excited by these very deep but high resolution optical images, and soon every ground and space-based telescope was investing huge amounts of observing time on the HDF-N and the surrounding Hubble Flanking Fields (HFF). It became clear that with the new MFFE systems installed on the WSRT, exploring large areas of the faint radio sky was now eminently possible. It struck me, that it would be interesting to see what this $\mu J y$ radio sky looked like on the largest angular scales - perhaps the WSRT could pick up extended objects missed (resolved) by the higher resolution VLA and e-MERLIN observations (Muxlow et al. 2005). The HDF-N (and HFFs) therefore became an obvious target for the WSRT - a nice opportunity to show case the improved performance of new MFFE systems. After submitting a proposal with Ger de Bruyn, Willem Baan and Richard Schilizzi, we were happy to receive 72 hours of observing time spread across the various array configurations in chunks of $6 \times 12$ hours.

During this period, I was also deeply involved in demonstrating and developing the concept of "wide-field VLBI". This had started in the late 1980 os while working with Dr. Richard Porcas on VLBI imaging of gravitationally lensed systems in which the lensed components could be separated by up to 10 arcseconds (or 10,000 synthesised beams). Richard had mentioned during my first visit to Bonn as a young PhD student, that one day it would be nice to image out the full field of view around each lensed systems, rather than target the lensed components individually via the tedious (and somewhat fraught) process of multiple-pass correlation. In particular, with multiple passes, the response of one lensed component could not be totally isolated from each other, leading to all sorts of problems on the shortest EVN baselines. In the early 1990s, I successfully applied wide-field techniques to several lensed systems (e.g. Garrett et al. 1994), mapping out large areas of the sky (at least in terms of VLBI) that encompassed the locations of all the lensed components in a single correlator pass. I extended these methods to the nearby nuclear star-forming galaxy M82, detecting several widely separated SNRs, located across the central region of the galaxy, a distance spanning several arcminutes on the sky (or $\sim 400,000$ beams!). Applying these wide-field techniques to other potential targets, I became almost evangelical about the promise of wide-field VLBI (Garrett et al. 1999), and bored people greatly about the evils of data averaging.

I think it's fair to say that not everyone fully shared my enthusiasm for this new approach! At that time, only a handful ofspecial targets could benefit (chiefly lenses, binary QSOs, and nearby galaxies), and the requirement to keep all the data in its raw, un-averaged form was a stretch for the compute and storage systems of the day.

The HDF-N would change all of that!

\section{WSRT data analysis}

It will come as no surprise to most readers, that Ger de Bruyn was very much in volved in the analysis of the WSRT HDF data. He was very keen to see if the new MFFEs were as good as the ASTRON engineers claimed. Ger was so familiar with analysing WSRT data, and adept with the manipulation of the home-grown Newstar software analysis package (see Chapter 6), that there was no need to spend too much time inspecting or editing data. The diagnostic output of the Newstar calibration and imaging tasks told Ger everything he needed to know about data quality. This approach also suited me quite well - experienced in handling the huge data sets generated by wide-field VLBI projects (a then staggering $120 \mathrm{~GB}$ !) I had long given up on the plodding approach to VLBI data processing that still absorbed most of my colleagues. Watching VLBI

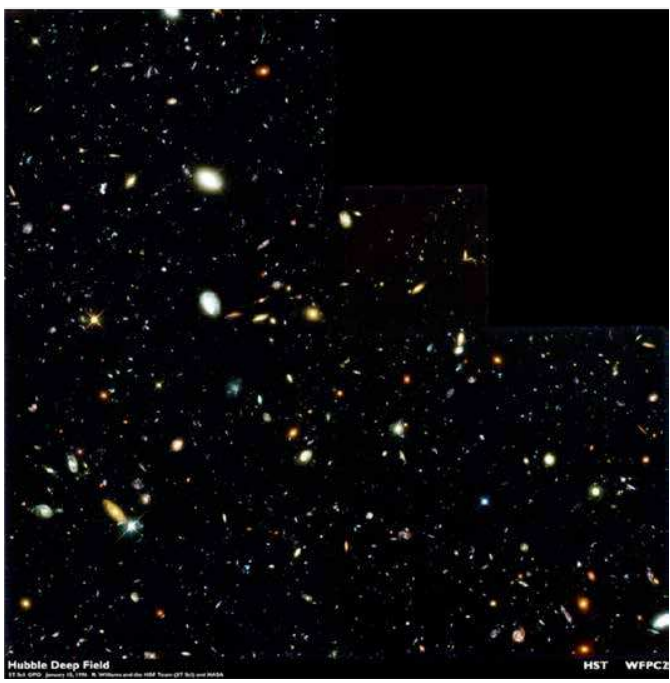

Figure 1: The Hubble Deep Field North (Williams et al. 1996) patch of sky no bigger than a grain
of sand held out of sand held out at arms length, yet encompassing
thousands of distant galaxies. 
astronomers editing individual visibilities by hand, and agonising over every point, was amusing but not very productive. It was naturally a challenge to keep up with Ger. Newstar was opaque to almost any normal mortal, save for a few astronomers that had used it in Dwingeloo, and perhaps the package's chief architect and advocate, Wim Brouw and Jan Noordam respectively.

In any case, the total intensity images produced by Newstar reached a noise level of $~ 9$ uJy (See the interior of Figure 2), and despite my best efforts, I could never get better than $10 \mu \mathrm{Jy}$ with AIPS. The Newstar images also looked better, and the linear polarisation images went deeper - to $7.5 \mu \mathrm{Jy}$ - this came as a surprise to me, and I assumed it was a dynamic range effect in stokesI but Ger rightly understood that this was a sure sign that the r.m.s. noise level of the total intensity maps included a contribution from not only thermal noise but also source confusion $(\sim 5 \mu \mathrm{Jy})$ adding in quadrature. Ger was greatly encouraged by this result - it basically meant that the sensitivity of the MFFEs, and the rest of the new WSRT system, worked pretty much "as advertised". Personally, I found it a bit disappointing - it seemed to me that the WSRT was now quickly confusion limited with maximum baselines of only $3 \mathrm{~km}$ and very sensitive receivers. This could only get "worse", as the total bandwidth expanded from

Figure 2: EVN detections of faint radio sources in the HDF and HFF frame the original WSRT contour map, superimposed on an CFHT I-band image of the field (Berger et al. 1998). Image courtesy of Jack Radcliffe.

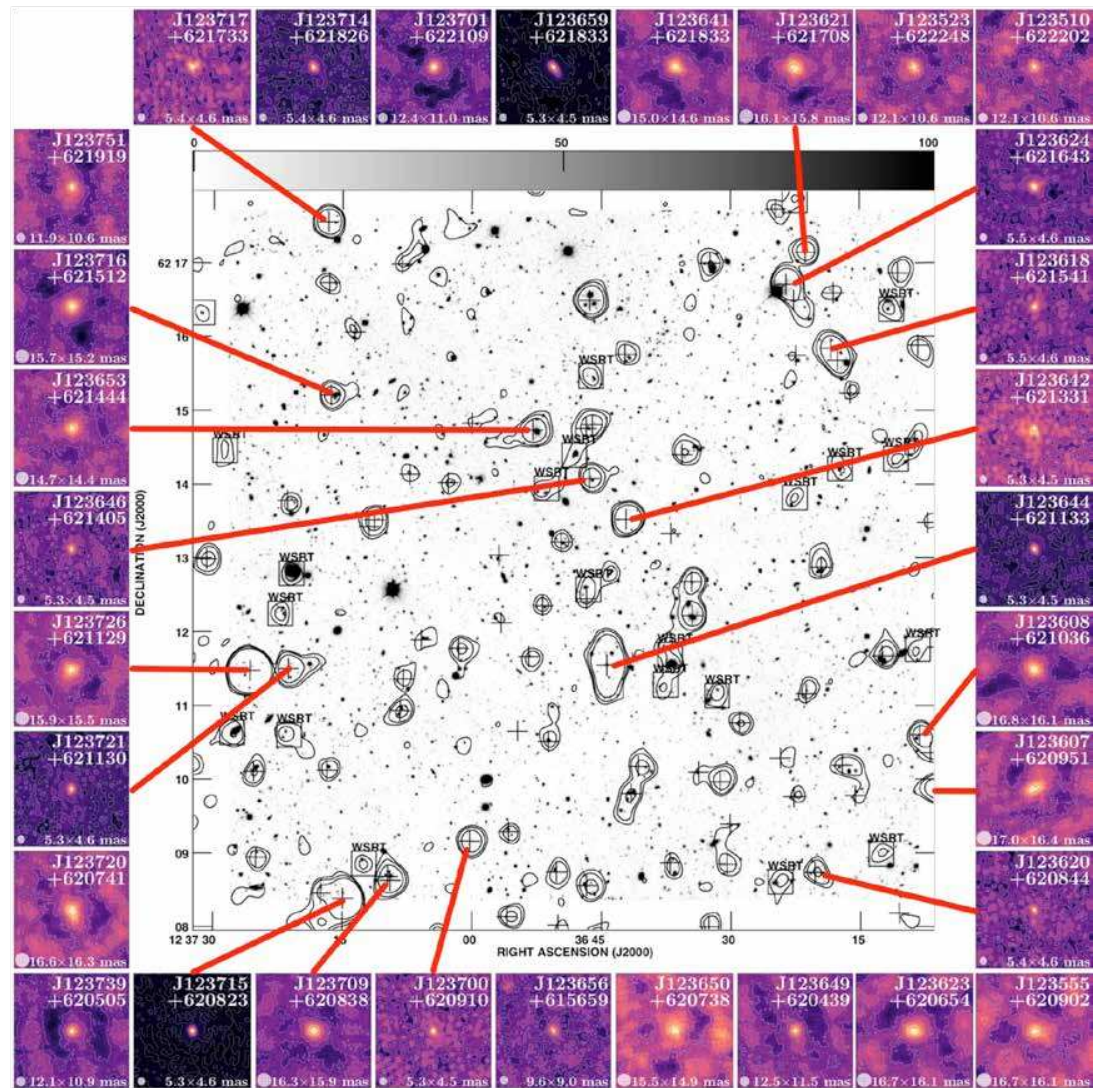

$80 \mathrm{MHz}$ to $160 \mathrm{MHz}$ with the introduction of the new IVC-DZB system later in the year. But looking to the future, it also meant that personally, the reduced "raw" sensitivity of the future Apertif L-band PAF system, was never to be a major issue for me.

I learned a lot from Ger during this period. My own background and expertise was very much rooted in high resolution radio astronomy, and in particular VLBI. First of all, I was shocked by the stability and quality of the WSRT data, as compared to VLBI! While the concept of filled apertures, redundancy and a 1-D East-West array seemed absolutely pre-historic to me (being raised, on a diet of sparse 2-D arrays, self-calibration and hybrid mapping), I noted that Ger made good use of these attributes, and by the end of the project I could appreciate their own merits. Some of these concepts have become of interest again, as the SKA1 with hundreds of antennas also offers superb aperture coverage. I was also surprised to find out that short baselines were not only a problem for VLBI but also the WSRT, with a mix of RFI, foreground emission and the response of the Sun being blamed. I was also very happy to see that the synthesised field-of view of the WSRT was so large that the map we produced was literally "teaming" with sources - they were all over the place, and to me this was how all radio astronomy should be, including VLBI. The fact that there was always enough sources in the field to self-calibrate the data was also pleasing, and I became more convinced than ever, that VLBI need not be any different in this respect, with in principle the primary beam being the only real limit to the field-of-view.

\section{WSRT Science Results}

The WSRT observations produced some fantastic images of the HDF and associated HFFs. Scientifically it was clear that the very deep but much higher resolution VLA and MERLIN observations were missing a substantial number of extended sources, providing a somewhat filtered view of the $\mu J y$ sky. The NRAO completion mumbled about confusion effects... but the upgraded WSRT detected many sources that the other arrays did not, and there was also evidence for AGN variability. Some of the new WSRT sources were also clearly associated with nearby star-forming galaxies, resolved across their disks in the higher resolution VLA maps. But perhaps the most important outcome was that a combination of the HDF-N $20 \mathrm{~cm}$ WSRT and $15 \mu \mathrm{m}$ ISO data demonstrated the surprising result that the FIR/MIR-Radio correlation held not only for galaxies in the local Universe but also for galaxies located at cosmological distances (Garrett 2002).

\section{EVN results}

The EVN observations of the HDF-N and the HFFs were made almost in parallel with the WSRT observations. They combined two techniques - a widefield analysis of the data, combined with the employment of phase-referencing (Beasley \& Conway 1995). Together this approach extended the coherence time of the array across the entire observing run, so that in principle the detection of 
submJy sources (and even $\mu J y$ sources) became possible across the entire field. The only question was what fraction of these faint sources would be compact on VLBI scales - at this point no one could be sure. It was therefore incredibly brave of the EVN Programme Committee (PC) to award a total of 32 hours of observing time dedicated to the HDF-N - the ultimate blank field (the HDF-N was specifically chosen to be devoid of bright radio sources). The observations were made in November 1999, correlated in Socorro and I analysed them on a working visit to Jodrell Bank in February 2000. I still remember the feeling of elation when the first three central sources popped up above the noise level, the faintest being only $180 \mu \mathrm{Jy}$. Figure 2 shows a more recent VLBI observation (Radcliffe et al. 2018) that achieves a central r.m.s. noise level of $5 \mu \mathrm{Jy}$, and detects over 30 sources.

I was incredibly excited by the EVN results - in addition to revealing the existence of very faint but compact sub-mJy and $\mu J y$ radio sources, it became clear from source brightness temperature arguments that the radio emission must arise from an AGN or be AGN dominated. This meant we could use VLBI to directly discriminate between emission generated by star formation and AGN processes in distant galaxies - a tricky business by other means. But I was actually more excited by the fact that at Lband we could now point the EVN anywhere on the sky, and detect multiple sources in the field - not only that but we later demonstrated that the accumulated response of all of these sources could permit self-calibration to be used (just like the WSRT) - even in a so-called "blank field" (e.g. Garrett et al. 2005, Radcliffe et al. 2017).

\section{Final thoughts}

My involvement in the WSRT observations of the HDF had many positive aspects. I got a chance to work with Ger, and to see at first hand a master craftsman at work. Although I was still working for JIVE at that time, leading a highly visible and successful WSRT project gave me the opportunity to engage with ASTRON staff and understand more about the organisation. It was an opportunity that eventually led to an interesting career path in which ASTRON would figure prominently.

\section{References}

Barger, A.J., Cowie, L.L., Trentham, N., Fulton, E., et al. 1998, Al 117, 102.

Beasley, A. J., \& Conway, J. E. 1995, in PASPC 82, ed. J. A. Zensus, P. J. Diamond, \& P. J. Napier, 328.

Noordam, J.E., The NEWSTAR Cookbook

Garrett, M.A., Calder, R.J., Porcas, R.W., et al. 1994, MNRAS, 270,457

Garrett, M. A., Porcas, R. W., Pedlar, et al. 1999, NewAR, 43, 521.

Garrett, M. A., de Bruyn, A. G., Giroletti, M., et al. 2000, A\&A, 361, L41. Garrett, M.A., Muxlow, T.W.B.,

Garrington, S.T., et al. 2001, A\&A, 366, L5.

Garrett 2002, A\&A, 581, L5.

Garrett, M.A., Wrobel. J.M. \& Morganti, R. 2005, ApJ, 619, 105.

Muxlow, T. W. B., Richards, A. M. S., Garrington, S. T., et al. 2005, MNRAS, 358, 1159.

Radcliffe, J.F., Garrett, M.A., Beswick, R.J., et al. 2017, A\&A, 587, A85.

Radcliffe, J.F., Garrett, M.A., Beswick, R.J., et al. 2018 (in press).

Williams, R. E., Blacker, B, Dickinson, M., et al. 1996, A), 112, 1335. 